\title{
Is unrelated donor or haploidentical hematopoietic transplantation preferred for patients with acute myeloid leukemia in remission?
}

\author{
Richard Champlin \\ University of Texas, MD Anderson Cancer Center, Houston, TX, USA \\ E-mail: RICHARD CHAMPLIN - rchampli@mdanderson.org
}

doi:10.3324/haematol.2019.239624

$I^{\text {nhes }}$ $\mathrm{n}$ this issue, Perales et al. ${ }^{1}$ address a controversy in hematopoietic stem cell transplantation: should an HLA-matched unrelated donor or haploidentical transplant be performed for patients who lack a matched sibling?

The preferred donor for allogeneic hematopoietic transplantation is an HLA-matched sibling. For several decades, an unrelated donor transplant has been considered the next option if an HLA-matched donor can be identified; survival after matched related and matched unrelated donor transplants has been similar in most studies.,3 A large worldwide network of unrelated donor registries has been established. Patients are most likely to have an HLA-matched donor among individuals with their same racial and ethnic origin. Matches can be found for about $60 \%$ of Caucasians, but only $30 \%$ for other ethnicities, primarily due to the racial composition of the existing donor registries. ${ }^{4}$ Some patients have a common HLA haplotype and have hundreds of matched donors, but at least half have rare or unique haplotypes and a matched unrelated donor cannot be found. Transplant outcomes have been best with younger unrelated donors, and males under the age of 40 are generally considered the preferred donors. ${ }^{5,6}$

The effective development of haploidentical hematopoietic stem cell transplantation (haploHSCT) has been a major therapeutic advance. ${ }^{7}$ The use of post-transplant cyclophosphamide, tacrolimus and mycophenolate mofetil has reduced the rates of acute and chronic graftversus-host disease (GvHD) with haploHSCT to below the levels reported with matched unrelated donor transplants, and with similar overall survival (OS). ${ }^{8}$ Haploidentical transplantation using this approach is now an established treatment option and is provided by transplant centers around the world.

The success of haploHSCT has led many to consider use of a haploidentical donor, even if a matched unrelated donor is potentially available. " The study reported by Perales et al. ${ }^{1}$ is a retrospective analysis conducted by the Center for Blood and Marrow Transplantation Research which compares the results of transplants from a young HLA-matched unrelated donor with haploidentical transplants from a sibling or child, in patients with acute myeloid leukemia (AML) in first or second complete remission. They analyzed 822 patients aged 50-75 years; 192 patients received haploidentical transplants and 631 patients had grafts from matched unrelated donors aged 18-40 years. Patients' and disease characteristics of the two groups were similar except unrelated donor recipients were more likely to have poor risk cytogenetics and to receive a myeloablative conditioning regimens. Post-transplant cyclophosphamide based GvHD prophylaxis was used in all patients receiving haploHSCT but none of the unrelated donor transplants. Five-year OS was 32\% and
$42 \%$ after haploHSCT and unrelated donor transplant, respectively $(P=0.04)$. Multivariable analysis showed improved survival and a lower risk of leukemia relapse after matched unrelated donor transplantation, with a similar risk of non-relapse mortality with either approach. They concluded that matched unrelated donor transplantation with donors younger than 40 years is preferred.

Large registry analyses have reported similar outcomes of haploidentical transplants with those from matched unrelated donors, ${ }^{8}$ but comparison of haploHSCT and unrelated donor transplants is complicated. Different preparative and GvHD prophylaxis regimens are generally used. Haploidentical transplants are more likely to be rejected than HLA-matched transplants, and most centers have used reduced intensity regimens in older adult patients, but intensified the preparative regimen to include low-dose total body irradiation (TBI) or thiotepa to prevent rejection.

The study by Perales et al. ${ }^{1}$ has several limitations due to imbalances in the treatment groups. The primary advantage for the unrelated donor group was a reduced rate of leukemia relapse. Myeloablative conditioning produces a lower rate of leukemia relapse than reduced intensity conditioning (RIC) in HLA-matched ${ }^{10,11}$ as well as haploidentical recipients with AML. ${ }^{12}$ The unrelated donor group predominantly received myeloablative conditioning, while the haploHSCT group mainly received a reduced intensity regimen with the cyclophosphamide fludarabine low-dose TBI regimen ${ }^{13}$ which is associated with a higher relapse rate than has been reported with other, more intensive, RIC regimens. ${ }^{14}$ Other retrospective studies have not reported higher relapse rates with haploHSCT compared to unrelated transplants. ${ }^{15,16}$ Use of a more intensive conditioning in the haploHSCT group may have potentially reduced relapse, but may also have increased non-relapse mortality.

The haploHSCT group had a lower rate of acute and chronic GvHD compared to the unrelated donor group. This is undoubtedly due to the use of post-transplant cyclophosphamide, tacrolimus and mycophenolate mofetil GvHD prophylaxis in the haploHSCT patients; this regimen is now being used for unrelated donor transplants as well, with improvement in control of GvHD. ${ }^{17-20}$ A formal comparison of post-transplant cyclophosphamide, tacrolimus and mycophenolate mofetil with tacrolimus methotrexate GvHD prophylaxis for unrelated donor transplants is in progress by the Blood and Marrow Transplant Clinical Trials Network.

\section{Advantages and disadvantages of haploidentical hematopoietic stem cell transplantation versus unrelated donor transplantation}

Haploidentical donors are identified by the initial family HLA typing so haploHSCT can be performed immedi- 
Table 1. Consideration for haploidentical versus unrelated donor transplants.

\section{Haploidentical transplants}

- Most (but not all) patients have a haploidentical donor

- Transplants can be performed immediately after family typing

- Transplant center controls the donor and all aspects of the transplant co-ordinates

- Preparative regimens are generally intensified with TBI or thiotepa to prevent rejection

- There is a low rate of severe acute GvHD and chronic GvHD with post transplant cyclophosphamide based GvHD prophylaxis

- Donor specific anti HLA antibodies may produce graft failure; must exclude patients with high titers

- Hemorrhagic cystitis and cardiac toxicity of cyclophosphamide may occur

- There is a longer time to hematologic recovery

\section{HLA matched unrelated donor transplants}

- HLA match can be identified for many patients, chance of success depends on race/ethnicity

- Established treatment modality; long track record

- Generally uses same preparative regimen and GvHD prophylaxis as matched related transplants

- Requires time for search process, coordination of the transplant with donor registries; patient s may deteriorate during this time; may need to accommodate delays for intercurrent patient or donor problems

- One can often avoid donor specific anti HLA antibodies

GvHD: graft-versus-host disease; TBI: total body irradiation.

ately, without a need to wait for an unrelated donor search. The process for performing the unrelated donor search to identify the donor and deliver the transplant typically takes 2-4 months, although donor registries are working to develop procedures that would shorten this process. This study involved AML patients in first or second remission, which can generally accommodate the time needed to organize an unrelated donor transplant. In more urgent clinical situations, like advanced acute leukemia, precarious patients may progress, clinically deteriorate, or acquire serious infections during the unrelated donor search and never receive a transplant. Often, the patient may develop medical complications that require the unrelated donor transplant to be rescheduled, which can be logistically challenging at short notice. If a prompt transplant is required and a matched unrelated donor is not immediately identified and available, it is appropriate to go forward with a haploHSCT.

Haploidentical transplants with post-transplant cyclophosphamide do have some special toxicities to consider. Hemorrhagic cystitis is a common complication and can be severe..$^{21}$ Cyclophosphamide can produce cardiac toxicity, particularly in those with pre-existing cardiac disease. The patient must have adequate renal function to safely tolerate post-transplant cyclophosphamide. Post-transplant cyclophosphamide does delay time to engraftment and hematologic recovery. Use of peripheral blood stem cells for haploidentical transplants accelerates hematopoietic recovery, but with an increased risk of GvHD.22

There are patients who lack an acceptable haploidentical donor, and an unrelated donor or cord blood is their only transplant option. These are typically older adults without healthy siblings or children. Cousins or other second-degree relatives who share a haplotype can be utilized for haploHSCT for these patients.

There are some advantages with unrelated donor transplants. It is a well established treatment modality with over 30 years of experience. A general principle of transplantation is that better matching is associated with intrinsically less alloreactivity and better transplant outcomes. One problem with haploidentical transplants is graft failure due to donor specific anti-HLA antibodies (DSA), particularly if positive by the C1q assay. ${ }^{23,24}$ Diffuse sensitization can be induced by blood transfusions, with high titer anti-HLA antibodies against a broad range of HLA antigens, primarily in parous female recipients. It is often impossible to identify a haploidentical donor without DSA for these individuals, and patients with high levels of DSA are appropriately excluded from trials of haploHSCT. Engraftment is not affected by antiHLA antibodies that are not donor specific. Often, an HLA-matched or one antigen mismatched unrelated donor can be identified, avoiding donor specific antibodies, in broadly sensitized patients. Note, unrelated donor transplants matched for HLA A, B, C, DR and DO are generally mismatched at DP, and anti-DP antibodies may be present which may lead to graft failure..$^{25}$

In conclusion, the study by Perales et al. ${ }^{1}$ reports that matched unrelated donor transplants with donors younger than 40 years of age is preferred to haploHSCT for patients with AML in complete remission, with improved survival and lower risk of relapse. That may be true for this relatively stable patient population using the preparative and GvHD prophylaxis regimens employed, but this conclusion may not hold for other patient populations where a prompt time to transplant is critical, or with alternative pre- and post-transplant treatment regimens. The ideal study would compare optimized versions of both haploidentical and unrelated donor transplants, and use "intention-to-treat" analysis including all patients for whom a transplant is intended from the time of initial HLA typing. The study by Perales et al. ${ }^{1}$ should give pause for thought, however, for those considering jumping to haploidentical transplants as a preferred approach in general. 


\section{References}

1. Perales MA, Tomlinson B, Zhang MJ, et al. Alternative donor transplantation for acute myeloid leukemia in patients aged $\geq 50$ years: young HLA-matched unrelated or haploidentical Donor? Haematologica. 2019;105(2):407-413.

2. Weisdorf DJ, Anasetti C, Antin JH, et al. Allogeneic bone marrow transplantation for chronic myelogenous leukemia: comparative analysis of unrelated versus matched sibling donor transplantation. Blood. 2002;99(6):1971-1977.

3. Gupta V, Tallman MS, He W, et al. Comparable survival after HLAwell-matched unrelated or matched sibling donor transplantation for acute myeloid leukemia in first remission with unfavorable cytogenetics at diagnosis. Blood. 2010;116(11):1839-1848.

4. Gragert L, Eapen M, Williams E, et al. HLA match likelihoods for hematopoietic stem-cell grafts in the U.S. registry. N Engl J Med. 2014;371(4):339-348.

5. Kollman C, Spellman SR, Zhang MJ, et al. The effect of donor characteristics on survival after unrelated donor transplantation for hematologic malignancy. Blood. 2016;127(2):260-267.

6. Shaw BE, Logan BR, Spellman SR, et al. Development of an Unrelated Donor Selection Score Predictive of Survival after HCT: Donor Age Matters Most. Biol Blood Marrow Transplant. 2018;24(5):1049-1056

7. Luznik L, O'Donnell PV, Symons HJ, et al. HLA-haploidentical bone marrow transplantation for hematologic malignancies using nonmyeloablative conditioning and high-dose, posttransplantation cyclophosphamide. Biol Blood Marrow Transplant. 2008;14(6):641650.

8. Ciurea SO, Zhang MJ, Bacigalupo AA, et al. Haploidentical transplant with posttransplant cyclophosphamide vs matched unrelated donor transplant for acute myeloid leukemia. Blood. 2015;126(8):1033-1040.

9. Ciurea SO, Bittencourt MCB, Milton DR, et al. Is a matched unrelated donor search needed for all allogeneic transplant candidates? Blood Adv. 2018;2(17):2254-2261.

10. Eapen M, Brazauskas R, Hemmer M, et al. Hematopoietic cell transplant for acute myeloid leukemia and myelodysplastic syndrome: conditioning regimen intensity. Blood Adv. 2018;2(16):2095-2103.

11. Scott BL, Pasquini MC, Logan BR, et al. Myeloablative Versus Reduced-Intensity Hematopoietic Cell Transplantation for Acute Myeloid Leukemia and Myelodysplastic Syndromes. J Clin Oncol. 2017;35(11):1154-1161.

12. Solomon SR, St Martin A, Shah NN, et al. Myeloablative vs reduced intensity T-cell-replete haploidentical transplantation for hematologic malignancy. Blood Adv. 2019;3(19):2836-2844.

13. Kasamon YL, Bolanos-Meade J, Prince GT, et al. Outcomes of Nonmyeloablative HLA-Haploidentical Blood or Marrow Transplantation With High-Dose Post-Transplantation
Cyclophosphamide in Older Adults. J Clin Oncol. 2015;33(28):3152 3161.

14. Ciurea SO, Shah MV, Saliba RM, et al. Haploidentical Transplantation for Older Patients with Acute Myeloid Leukemia and Myelodysplastic Syndrome. Biol Blood Marrow Transplant. 2018;24(6):1232-1236.

15. Bashey ZA, Zhang X, Brown S, et al. Comparison of outcomes following transplantation with T-replete HLA-haploidentical donors using post-transplant cyclophosphamide to matched related and unrelated donors for patients with AML and MDS aged 60 years or older. Bone Marrow Transplant. 2018;53(6):756-763.

16. Nagler A, Ruggeri A. Haploidentical stem cell transplantation (HaploSCT) for patients with acute leukemia-an update on behalf of the ALWP of the EBMT. Bone Marrow Transplant. 2019;54(Suppl 2):713-718.

17. Mielcarek M, Furlong T, O'Donnell PV, et al. Posttransplantation cyclophosphamide for prevention of graft-versus-host disease after HLA-matched mobilized blood cell transplantation. Blood. 2016;127(11):1502-1508.

18. El Fakih R, Hashmi SK, Ciurea SO, Luznik L, Gale RP, Aljurf M. Posttransplant cyclophosphamide use in matched HLA donors: a review of literature and future application. Bone Marrow Transplant. 2019 May 14. [Epub ahead of print]

19. Mehta RS, Saliba RM, Chen J, et al. Post-transplantation cyclophosphamide versus conventional graft-versus-host disease prophylaxis in mismatched unrelated donor haematopoietic cell transplantation. Br J Haematol. 2016;173(3):444-455.

20. Rashidi A, Hamadani M, Zhang MJ, et al. Outcomes of haploidentical vs matched sibling transplantation for acute myeloid leukemia in first complete remission. Blood Adv. 2019;3(12):1826-1836.

21. Copelan OR, Sanikommu SR, Trivedi JS, et al. Higher Incidence of Hemorrhagic Cystitis Following Haploidentical Related Donor Transplantation Compared with Matched Related Donor Transplantation. Biol Blood Marrow Transplant. 2019;25(4):785-790.

22. Bashey A, Zhang MJ, McCurdy SR, et al. Mobilized Peripheral Blood Stem Cells Versus Unstimulated Bone Marrow As a Graft Source for T-Cell-Replete Haploidentical Donor Transplantation Using PostTransplant Cyclophosphamide. J Clin Oncol. 2017;35(26):30023009.

23. Ciurea SO, Thall PF, Milton DR, et al. Complement-Binding DonorSpecific Anti-HLA Antibodies and Risk of Primary Graft Failure in Hematopoietic Stem Cell Transplantation. Biol Blood Marrow Transplant. 2015;21(8):1392-1398.

24. Ciurea SO, de Lima M, Cano P, et al. High risk of graft failure in patients with anti-HLA antibodies undergoing haploidentical stemcell transplantation. Transplantation. 2009;88(8):1019-1024.

25. Spellman S, Bray R, Rosen-Bronson S, et al. The detection of donordirected, HLA-specific alloantibodies in recipients of unrelated hematopoietic cell transplantation is predictive of graft failure. Blood. 2010;115(13):2704-2708. 